\title{
ON THE GENERALIZED VARIATION AND GENERALIZED WEAK VARIATION OF MAPS WITH VALUES IN METRIC LINEAR SPACES
}

\author{
RoBERT DROZDOWSKI
}

\begin{abstract}
In this paper, the class of maps (with values in a metric linear space) of a bounded generalized variation (bounded generalized weak variation) is described. Connections between those kinds of spaces are investigated.
\end{abstract}

\section{Introduction}

In the literature one can find many generalizations of a well-known notion of variation of a real valued function of one variable. For example, D. W a t e r m a $\mathrm{n}$ introduced an idea of the function of bounded $\lambda$-variation in [5], an additive map defined on $\sigma$-algebra of subsets of $X$ was considered by S. S a k s in $[\underline{8}$ and B. Th o m s o n studied the class of interval functions of bounded $S$-variation 9 . Another kind generalizations is $\varphi$-variation of a map having values in a metric space and defined on a subset of $\mathbb{R}$; in particular, it was considered in [2]. One can also find generalizations of ideas introduced by $\mathrm{D}$. W a t e r $\mathrm{m}$ a $\mathrm{n}$ and B. Thomson in [3].

In this paper we will introduce a concept of generalized variation in the restricted sense (weak generalized variation in the restricted sense) for a map defined on a nonempty subset $E$ of $\mathbb{R}$ and having values in a metric space $(X, d)$, which is a more general notion to those considered in [1].

If $(X, d)$ is a metric space then $B^{\circ}(x, \varepsilon), \bar{B}(x, \varepsilon)$ will denote the open ball with the center $x$ from $X$ and positive radius $\varepsilon$, and the closed ball with the center $x$ in $X$ and positive radius $\varepsilon>0$, respectively. Under additional assumption of $X$ that is a linear space over a field $\Phi$ with operation of addition " + " and multiplying by scalars ".", it is said that $d$ is a translation invariant metric if $d(x+z, y+z)=$ $d(x, y)$ for each $x, y$ and $z$ from $X$ (terminology is taken from [7]). 


\section{ROBERT DROZDOWSKI}

Let us emphasize that any linear space will always be considered over the field of real or complex numbers, i.e., $\Phi=\mathbb{R}$ or $\Phi=\mathbb{C}$. Moreover, if $X$ is a linear space over $\Phi$, then for a subset $A$ and a subset $B$ of $X$, the symbol $A+B$ and $\lambda A(\lambda \in \Phi)$ will stand to denote the sets of the form:

$$
A+B=\{a+b: a \in A \wedge b \in B\}
$$

and

$$
\lambda A=\{\lambda A: a \in A\} .
$$

If $f: E \rightarrow X$ is a map, where $E$ is a nonempty subset of $\mathbb{R}$ and $(X, d)$ is a metric space, then, for a subset $A$ of $E$, the quantity

$$
\sup \{(f(x), f(y)): x \in A, y \in A\}
$$

will be said to be $d$-oscillation of $f$ on $A$ and will be denoted as $\omega_{d}(f, A)$. In a special case, if $A=\emptyset$, we write $\omega_{d}(f, A)=0$.

Throughout this paper, the symbol $\mathcal{R}(E)$ will denote the class of all finite collections of closed and nonoverlapping intervals $\left\{I_{1}, \ldots, I_{n}\right\}$ such that

$$
I_{i} \cap E \neq \emptyset \quad \text { if } \quad i \in\{1, \ldots, n\}
$$

and

$$
\bigcup_{i=1}^{n}\left(I_{i} \cap E\right)=E .
$$

Definition 1.1. Let $(X, d)$ be a metric space and $E$ be a nonempty subset of $\mathbb{R}$. It will be said that a map $f: E \rightarrow X$ has a bounded variation in the restricted sense if the quantity

$$
\sup \left\{\sum_{i=1}^{n} \omega_{d}\left(f, I_{i} \cap E\right):\left\{I_{i}: i=1, \ldots, n\right\} \in \mathcal{R}(E)\right\}
$$

is finite. The generalized variation in the restricted sense of $f$ on $E$ will be denoted by $V_{d}(f, E)$.

We say that a map $f: E \rightarrow X$ has bounded generalized variation in the restricted sense if $E$ can be represented as a countable union of closed sets $\left\{E_{i}: i \in \mathbb{N}\right\}$ such that $V_{d}\left(f, E_{i}\right)<\infty$ for each $i \in \mathbb{N}$.

Definition 1.2. Let $(X, d)$ be a linear topological space, $X^{\star}$ be dual space to $X$, and $\Gamma$ be a nonempty subfamily of $X^{\star}$. It is said that a map $f: E \rightarrow X$ has a bounded weak variation in the restricted sense on $E$ with respect to $\Gamma$, if for each $\Lambda$ from $\Gamma$, the composition $\Lambda \circ f$ has a bounded variation in the restricted sense on $E$.

A map $f: E \rightarrow X$ has a bounded generalized weak variation in the restricted sense with respect to $\Gamma$, if for each $\Lambda \in \Gamma$, the composite function $\Lambda \circ f$ has a bounded generalized variation in the restricted sense on $E$. 


\section{ON THE GENERALIZED VARIATION}

From now on, we will use the following denotations:

(1) $d V B G$ - the class of maps of a bounded variation in the restricted sense on $E$ with respect to the metric $d$ in $X$;

(2) $d V B G_{\star}$ - the class of maps of a bounded generalized variation in the restricted sense on $E$ with respect to the metric $d$ in $X$;

(3) $\Gamma W B G(X)$ - the class of maps of a bounded weak variation in the restricted sense on $E$ with respect to the subfamily $\Gamma$ of $X^{\star}$;

(4) $\Gamma W B G_{\star}(X)$ - the class of maps of a bounded generalized weak variation in the restricted sense on $E$ with respect to the subfamily $\Gamma$ of $X^{\star}$.

Let us remark that if $X=\mathbb{R}$ and $d_{n}$ is Euclidean metric in $X$, then $d_{n} V B G$, $d_{n} V B G_{\star}$ are the classes of functions of a bounded variation in the restricted sense on $E$, and the classes of functions of a bounded generalized variation in the restricted sense on $E$, respectively [1. Instead of denotations $d_{n} V B G$, $d_{n} V B G_{\star}$, we will use the symbols $V B G, V B G_{\star}$, respectively.

\section{General properties}

At the beginning, some basic properties of maps of a bounded generalized variation in the restricted sense and a bounded generalized weak variation in the restricted sense will be proved. It is not difficult to see that, if $(X, d)$ is a metric space, $E_{1} \subset E$ and $E_{1}, E$ are subsets of $\mathbb{R}$, then for a map $f: E \rightarrow \mathbb{R}$ the inequality

$$
V_{d}\left(f, E_{1}\right) \leq V_{d}(f, E)
$$

holds. To simplify our considerations, from now on, it will be always assumed that $X$ is a real or complex metric linear space $[6]$.

Theorem 2.1. Let $(X, d)$ be a metric space, where $d$ is a translation invariant metric. If $f_{1}, f_{2} \in d V B G$, then $f_{1}+f_{2} \in d V B G$.

P r o of. Let us assume that $f_{1}, f_{2}$ belong to $d V B G$ and take $\left\{I_{1}, \ldots, I_{n}\right\}$ from $\mathcal{R}(E)$. For each $x, y$ from $I_{i} \cap E$, we have

$$
\begin{aligned}
d\left(\left(f_{1}+f_{2}\right)(x),\left(f_{1}+f_{2}\right)(y)\right) & =d\left(f_{1}(x)+f_{2}(x), f_{1}(y)+f_{2}(y)\right) \\
& =d\left(f_{1}(x)-f_{1}(y), f_{2}(x)-f_{2}(y)\right) \\
& \leq d\left(f_{1}(x)-f_{1}(y), 0\right)+d\left(f_{2}(x)-f_{2}(y), 0\right) \\
& =d\left(f_{1}(x), f_{1}(y)\right)+d\left(f_{2}(x), f_{2}(y)\right) \\
& \leq \omega_{d}\left(f_{1}, I_{i} \cap E\right)+\omega_{d}\left(f_{2}, I_{i} \cap E\right),
\end{aligned}
$$

whence

$$
\omega_{d}\left(f_{1}+f_{2}, I_{i} \cap E\right) \leq \omega_{d}\left(f_{1}, I_{i} \cap E\right)+\omega_{d}\left(f_{2}, I_{i} \cap E\right)
$$




\section{ROBERT DROZDOWSKI}

if $i \in\{1, \ldots, n\}$ and, consequently,

$$
V_{d}\left(f_{1}+f_{2}, E\right) \leq V_{d}\left(f_{1}, E\right)+V_{d}\left(f_{2}, E\right) .
$$

From the assumption and the above inequality, we infer that $f_{1}+f_{2} \in d V B G$.

A subset $E$ of a metric space is called to be bounded if the diameter of $E$ is finite. Apart from that, in the literature also there appears a definition of bounded subsets in linear topological spaces. According to [7, a subset $E$ of linear topological space is said to be bounded if for each neighborhood $V$ of 0 from $X$ there exists a real positive number $s$ such that $E \subset t V$ for each $t>s$.

To distinguish those two notions, we say that a set $E$ is bounded in the usual sense if it has the finite diameter (in metric space) and a subset $E$ is bounded if it is bounded in the sense given in [7].

Let us remark that in the space $\Phi$ with the Euclidean metric definitions of "bounded subset in the usual sense" and "bounded subset" coincide.

Similarly, a map $f$ from $E$ into a metric linear space $X$ is said to be bounded in the usual sense if $f(E)$ is bounded in the usual sense and it is called to be bounded if $f(E)$ is bounded. Moreover, if $X$ is a linear topological space, then a map $f: E \rightarrow X$ is said to be weakly bounded, if for each $\Lambda \in X^{\star}$, the set $\Lambda(f(E))$ is bounded.

TheOREM 2.2. If $(X, d)$ is a metric space, $E \subset \mathbb{R}$ and $f \in d V B G$, then $f$ is bounded in the usual sense.

P r o of. There exists a partition $\left\{I_{1}, \ldots, I_{n}\right\}$ from $\mathcal{R}(E)$ such that

$$
\sum_{i=1}^{n} \omega_{d}\left(f, I_{i} \cap E\right)<V_{d}(f, E)+1 .
$$

Without lost of generality, one can assume that

$$
\sup \left\{x: x \in I_{i}\right\} \leq \inf \left\{x: x \in I_{i+1}\right\},
$$

where $i \in\{1, \ldots, n-1\}$. If $x$ and $y$ belong to $E$, then $x \in I_{i} \cap E, y \in I_{j} \cap E$ for some $i, j \in\{1, \ldots, n\}$. If $i \leq j$, then:

$$
\begin{aligned}
d(f(x), f(y)) \leq & d\left(f(x), f\left(x_{i+1}\right)\right)+d\left(f\left(x_{i+1}\right), f\left(x_{i+2}\right)\right)+\ldots \\
& \ldots+d\left(f\left(x_{j-2}\right), f\left(x_{j-1}\right)\right)+d\left(f\left(x_{j-1}\right), f(y)\right) \\
\leq & \omega_{d}\left(f, I_{i} \cap E\right)+\omega_{d}\left(f, I_{i+1} \cap E\right)+\ldots \\
& \ldots+\omega_{d}\left(f, I_{j-1} \cap E\right)+\omega_{d}\left(f, I_{j} \cap E\right) \\
\leq & V_{d}(f, E)+1 .
\end{aligned}
$$

Similarly, one can prove that

$$
d(f(x), f(y)) \leq V_{d}(f, E)+1 \quad \text { if } \quad i \geq j .
$$


Consequently, the diameter of $f(E)$ is finite since $x, y$ have been chosen in arbitrary way, which means that $f$ is bounded in the usual sense.

THEOREM 2.3. Let us assume that:

(1) $(X, d)$ is a metric space and $d$ is a translation invariant metric in $X$;

(2) if $U$ is a bounded subset in the usual sense of $X$, then there exists a positive real number $M_{U}$ such that for each $x$ from $U$ and each $|\beta| \leq 1$ from $\Phi$ the inequality $d(\beta x, 0) \leq M_{U} d(x, 0)$ holds.

Then $\alpha f \in d V B G$ for each $\alpha$ from $\Phi$ and each $f \in d V B G$.

Proof. Let $f \in d V B G$. Then $f(E)-f(E)$ is a bounded subset of $X$ in the usual sense, since, by previous theorem, $f(E)$ is a bounded subset of $X$ in the usual sense. Let us consider a closed interval $I$ such that $I \cap E \neq \emptyset$ and $\alpha$ from $\Phi$, where $\alpha=u+i v(u, v \in \mathbb{R})$. If $x, y$ belong to $I \cap E$, then

$$
\begin{aligned}
d((\alpha f)(x),(\alpha f)(y))= & d(u f(x)+i v f(x), u f(y)+i v f(y)) \\
= & d(u f(x)-u f(y), i v f(y)-i v f(x)) \\
\leq & d(u(f(x)-f(y)), 0)+d(0, i v(f(y)-f(x))) \\
\leq & d([u](f(x)-f(y)), 0)+d((u-[u])(f(x)-f(y)), 0) \\
& +d(i[v](f(y)-f(x)), 0)+d(i(v-[v])(f(y)-f(x)), 0) \\
\leq & |[u]| d(f(x), f(y))+M d(f(x), f(y)) \\
& +M|[v]| d(f(x), f(y))+M d(f(x), f(y)) \\
\leq & (|[u]|+M(2+|[v]|)) \omega_{d}(f, I \cap E),
\end{aligned}
$$

where the symbol $[x]$ denotes entire of $x$.

It follows that for a partition $\left\{I_{1}, \ldots, I_{n}\right\}$ from $\mathcal{R}(E)$ we have

$$
\begin{aligned}
\sum_{i=1}^{n} \omega_{d}\left(\alpha f, I_{i} \cap E\right) & \leq(|[u]|+M(2+|[v]|)) \cdot \sum_{i=1}^{n} \omega_{d}\left(f, I_{i} \cap E\right) \\
& \leq(|[u]|+M(2+|[v]|)) \cdot V_{d}(f, E)
\end{aligned}
$$

whence

$$
V_{d}(\alpha f, E) \leq(|[u]|+M(2+|[v]|)) \cdot V_{d}(f, E) .
$$

It means that $\alpha f \in d V B G$.

The following example shows that the condition (2) of the above theorem is essential.

EXAMPLE 2.1. Let

$$
E_{1}=\left\{\frac{1}{2^{2 n}}: n \in \mathbb{N}\right\} \quad \text { and } \quad E_{2}=\left\{\frac{1}{2^{2 n-1}}: n \in \mathbb{N}\right\} .
$$


Let $g: E_{1} \longrightarrow \mathbb{R}$ satisfy the following conditions:

(1) $g\left(\frac{1}{2^{2 n}}\right)>g\left(\frac{1}{2^{2 n+2}}\right)>0$ for all $n \in \mathbb{N}$;

(2) $\lim _{n \rightarrow \infty} g\left(\frac{1}{2^{2 n}}\right)=0$;

(3) $g$ has bounded variation in the restricted sense on $E_{1}$.

Let $h: E_{2} \longrightarrow \mathbb{R}$ satisfy the following conditions:

(1) $0<h\left(\frac{1}{2^{2 n-1}}\right)<h\left(\frac{1}{2^{2 n+1}}\right)$ for all $n \in \mathbb{N}$;

(2) $\lim _{n \rightarrow \infty} h\left(\frac{1}{2^{2 n-1}}\right)=\infty$;

(3) $h$ does not have a bounded variation in the restricted on $E_{2}$.

Additionally, let us assume that

$$
\left\{g(x): x \in E_{1}\right\} \cap\left\{h(x): x \in E_{2}\right\}=\emptyset .
$$

Lastly, we define a function $u: \mathbb{R} \backslash\left(E_{1} \cup E_{2}\right) \longrightarrow \mathbb{R}$ such that $u$ is one to one, $u(0)=0$ and

$$
u\left(\mathbb{R} \backslash\left(E_{1} \cup E_{2}\right)\right) \cap\left(g\left(E_{1}\right) \cup h\left(E_{2}\right)\right)=\emptyset .
$$

The function $k: \mathbb{R} \rightarrow \mathbb{R}$ defined as follows

$$
k(x)= \begin{cases}g(x) & \text { if } x \in E_{1} \\ h(x) & \text { if } x \in E_{2}, \\ u(x) & \text { if } x \in \mathbb{R} \backslash\left(E_{1} \cup E_{2}\right)\end{cases}
$$

is one to one function from $\mathbb{R}$ to $\mathbb{R}$, so the map

$$
d(x, y)=|k(x)-k(y)| \quad x, y \in \mathbb{R}
$$

is a metric in $\mathbb{R}$.

Now, let us consider the space $\mathbb{R}$ with the metric $d$ and $E=\left\{\frac{1}{n}: n \in \mathbb{N}\right\}$, and $f: E \rightarrow \mathbb{R}$, for which $f\left(\frac{1}{n}\right)=\frac{1}{2^{2 n}}$, if $n \in \mathbb{N}$.

Then, one can observe that $V_{d}(f, E)=V\left(g, E_{1}\right)<\infty$, so $f$ has a bounded variation in the restricted sense on $E$.

On the other hand, $V_{d}\left(\frac{1}{2} f, E\right)=V\left(h, E_{2}\right)=\infty$ and, consequently, $\frac{1}{2} f \notin$ $d V B G$.

TheOREM 2.4. If $(X, d)$ is a metric space where $d$ is a translation invariant metric then

$$
f_{1}+f_{2} \in d V B G_{\star} \quad \text { for each } f_{1}, f_{2} \quad \text { from } d V B G_{\star} .
$$

Proof. If $f_{1} \in d V B G_{\star}$, then $E$ can be represented in the form $E=\bigcup_{i=1}^{\infty} H_{i}$, where $V_{d}\left(f_{1}, H_{i}\right)$ is finite and $H_{i}$ are closed for each $i$ from $\mathbb{N}$. For same reason, $E$ can be represented as $E=\bigcup_{i=1}^{\infty} G_{i}$, where $V_{d}\left(f_{2}, G_{i}\right)$ is finite and $G_{i}$ are closed for all $i$ from $\mathbb{N}$. Thus

$$
E=\bigcup_{i=1}^{\infty} \bigcup_{j=1}^{\infty}\left(H_{i} \cap G_{j}\right),
$$




\section{ON THE GENERALIZED VARIATION}

$$
\begin{aligned}
& V_{d}\left(f_{1}, H_{i} \cap G_{j}\right) \leq V_{d}\left(f_{1}, H_{i}\right)<\infty, \\
& V_{d}\left(f_{2}, H_{i} \cap G_{j}\right) \leq V_{d}\left(f_{2}, G_{j}\right)<\infty .
\end{aligned}
$$

It follows from the above relations that $E$ is a countable union of closed sets $H_{i} \cap G_{j}$ and

$$
V_{d}\left(f_{1}+f_{2}, H_{i} \cap G_{j}\right) \leq V_{d}\left(f_{1}, H_{i} \cap G_{j}\right)+V_{d}\left(f_{2}, H_{i} \cap G_{j}\right)<\infty
$$

for each $i, j$ from $\mathbb{N}$. By Definition 1.1 $f_{1}+f_{2}$ has a bounded generalized variation in the restricted sense on $E$.

Applying Theorem 2.3 and the method analogous to that of the proof of Theorem 2.4, one can prove the following

TheOREM 2.5. If the assumptions from Theorem 2.3 are satisfied, then $\alpha f \in$ $d V B G_{\star}$, where $\alpha \in \Phi$ and $f \in d V B G_{\star}$.

One can observe that, under the assumptions of Theorem 2.3, $d V B G$ and $d V B G_{\star}$ are linear spaces over the field $\Phi$.

There is a less complicated problem to conclude that

$$
\Gamma W B G(X) \text { and } \Gamma W B G_{\star}(X)
$$

are linear spaces over $\Phi$.

TheOREM 2.6. If $X$ is a linear topological space, $\Gamma$ is a nonempty subfamily of $X^{\star}$, then

$$
f_{1}+f_{2} \in \Gamma W B G(X) \quad \text { and } \quad \alpha f_{1} \in \Gamma W B G(X)
$$

for $\alpha$ from $\Phi$ and $f_{1}, f_{2}$ from $\Gamma W B G(X)$.

P r o o f. If $f_{1}, f_{2}$ belong to $\Gamma W B G(X)$ and $\left\{I_{1}, \ldots, I_{n}\right\}$ is a partition from $\mathcal{R}(E)$, then $\Lambda \circ f_{1}$ and $\Lambda \circ f_{2}$ belong to $V B G$. For $x, y$ from $I_{i}$ we have:

$$
\begin{aligned}
\left|\Lambda\left(\left(f_{1}+f_{2}\right)(x)\right)-\Lambda\left(\left(f_{1}+f_{2}\right)(y)\right)\right| & =\left|\left(\Lambda f_{1}(x)-\Lambda f_{2}(y)\right)+\left(\Lambda f_{1}(y)-\Lambda f_{2}(y)\right)\right| \\
& \leq\left|\Lambda f_{1}(x)-\Lambda f_{2}(y)\right|+\left|\Lambda f_{1}(y)-\Lambda f_{2}(y)\right| \\
& \leq \omega\left(\Lambda \circ f_{1}, I_{i} \cap E\right)+\omega\left(\Lambda \circ f_{2}, I_{i} \cap E\right)
\end{aligned}
$$

hence

$$
\omega\left(\Lambda \circ\left(f_{1}+f_{2}\right), I_{i} \cap E\right) \leq \omega\left(\Lambda \circ f_{1}, I_{i} \cap E\right)+\omega\left(\Lambda \circ f_{2}, I_{i} \cap E\right) .
$$

In consequence,

$$
\begin{aligned}
\sum_{i=1}^{n} \omega\left(\Lambda \circ\left(f_{1}+f_{2}\right), I_{i} \cap E\right) & \leq \sum_{i=1}^{n} \omega\left(\Lambda \circ f_{1}, I_{i} \cap E\right)+\sum_{i=1}^{n} \omega\left(\Lambda \circ f_{2}, I_{i} \cap E\right) \\
& \leq V\left(\Lambda \circ f_{1}, E\right)+V\left(\Lambda \circ f_{2}, E\right)
\end{aligned}
$$

which implies that

$$
V\left(\Lambda \circ\left(f_{1}+f_{2}\right), E\right)
$$


is finite for each linear functional $\Lambda$ from $\Gamma$. Finally,

$$
f_{1}+f_{2} \in \Gamma W B G(X) .
$$

Analogously, one can prove that $\alpha f \in \Gamma W B G(X)$ whenever $\alpha \in \Phi$ and $f \in \Gamma W B G(X)$.

Theorem 2.7. Let $X$ be a linear topological space, $E \subset \mathbb{R}, \Gamma=X^{\star}$ and $f \in$ $\Gamma W B G(X)$. Then $f$ is weakly bounded.

Proof. If $f \in \Gamma W B G(X)$ and $\Lambda \in X^{\star}$, then $\Lambda \circ f$ belongs to $V B G$. By Theorem 2.2, $\Lambda \circ f$ is bounded in the usual sense in $\Phi$, so it is bounded. Hence, $f$ is weakly bounded, since $\Lambda$ has chosen arbitrarily.

Applying Theorem 2.6 and 2.7, one can prove the following

TheOREM 2.8. If $X$ is a linear topological space, $\Gamma$ is a nonempty subset of $X^{\star}$, then

$$
f_{1}+f_{2} \in W B G_{\star}(X) \text { and } \alpha f_{1} \in W B G_{\star}(X)
$$

for each $\alpha$ from $\Phi$ and $f_{1}, f_{2}$ from $W B G_{\star}(X)$.

\section{Spaces $d \widetilde{V} B G$ and $\Gamma \widetilde{W} B G$}

Let us consider a relation " $\sim$ " in $d V B G$ defined in the following way:

$$
f \sim g \Longleftrightarrow V_{d}(f-g, E)=0
$$

for each $f, g \in d V B G$. One can verify that " $\sim$ " is an equivalence relation in $d V B G$. The set of all equivalence classes determined by " $"$ will be denoted by $d \widetilde{V} B G$. It can be proved that $d \widetilde{V} B G$ is a metric space equipped with the metric

$$
\widetilde{\varrho}(\widetilde{f}, \widetilde{g})=V_{d}(f-g, E) \quad(\tilde{f}, \widetilde{g} \in d \widetilde{V} B G)
$$

which is also a translation invariant. It is also not difficult to observe that, under the assumptions of Theorem 2.3. $d \widetilde{V} B G$ is a linear space over $\Phi$, where the operation of addition and multiplying by scalars are defined in a standard way. However, under some additional assumptions of metric $d$, the following can be proved

Theorem 3.1. Let $(X, d)$ be a metric space over $\Phi$, and d a translation invariant metric in $X$ satisfying the condition

- there exists a bounded and real valued function $\psi$ defined on $\{\alpha \in \Phi:|\alpha| \leq 1\}$ such that $\lim _{\alpha \rightarrow 0} \psi(\alpha)=0$ and if $\alpha$ belongs to $\{\alpha \in \Phi:|\alpha| \leq 1\}$ and $x$ in $X$, then the inequality $d(\alpha x, 0) \leq \psi(\alpha) d(x, 0)$ holds.

Then $d \widetilde{V} B G$ is a linear topological space over $\Phi$. 


\section{ON THE GENERALIZED VARIATION}

P r o o f. Addition is a continuous operation, since $(d \widetilde{V} B G, \widetilde{\varrho})$ is a metric space. It is sufficient to show that multiplication by scalars from $\Phi$ is a continuous operation; it means that the function $F: \Phi \times d \widetilde{V} B G \rightarrow d \widetilde{V} B G$, where

$$
F(\alpha, f)=\alpha f \quad(\alpha \in \Phi, f \in d \widetilde{V} B G)
$$

is continuous. Let us choose a sequence $\left(\alpha_{n}\right)_{n=1}^{\infty}$ of scalars and a sequence $\left(f_{n}\right)_{n=1}^{\infty}$ of elements of $d \widetilde{V} B G$ which are convergent to $\alpha$ and $f$, respectively. There exists a positive number $M$ such that:

$$
V_{d}\left(\alpha\left(f_{n}-f\right), E\right) \leq M V_{d}\left(f_{n}-f, E\right),
$$

whence $\lim _{n \rightarrow \infty} V_{d}\left(\alpha\left(f_{n}-f\right), E\right)=0$. On the other hand,

$$
V_{d}\left(\left(\alpha_{n}-\alpha\right) f, E\right) \leq \psi\left(\alpha_{n}-\alpha\right) V_{d}(f, E)
$$

for sufficiently large $n$ and, in consequence, $\lim _{n \rightarrow \infty} V_{d}\left(\alpha_{n}-\alpha, E\right)=0$. Since

$$
\widetilde{\varrho}\left(\alpha_{n} f_{n}, \alpha f\right) \leq \widetilde{\varrho}\left(\alpha f_{n}, \alpha f\right)+\widetilde{\varrho}\left(\alpha_{n} f, \alpha f\right),
$$

so $\lim _{n \rightarrow \infty} \widetilde{\varrho}\left(\alpha_{n} f_{n}, \alpha f\right)=0$ and the proof is complete.

LEMMA 3.1. Let us assume that $(X, d)$ is a metric space with translation invariant metric $d$, and $\left(f_{n}\right)_{n=1}^{\infty}$ converges uniformly to a map $f$ with respect to metric $d$, where $f_{n}, f: E \rightarrow X$. Then

$$
\lim _{n \rightarrow \infty} \omega_{d}\left(f_{n}, I \cap E\right)=\omega_{d}(f, I \cap E)
$$

for each closed interval $I$.

Proof. If $I$ is an interval for which $I \cap E \neq \emptyset$, then for $\varepsilon>0$ there exists $n_{0} \in \mathbb{N}$ such that if $n \geq n_{0}$ and $x \in I \cap E$, then $d\left(f_{n}(x), f(x)\right)<\frac{\varepsilon}{4}$. For $x, y$ from $I \cap E$ we get:

$$
\begin{aligned}
d(f(x), f(y)) & \leq d\left(f_{n}(x), f(x)\right)+d\left(f_{n}(y), f(y)\right)+d\left(f_{n}(x), f_{n}(y)\right) \\
& <\frac{\varepsilon}{2}+\omega_{d}\left(f_{n}, I \cap E\right) .
\end{aligned}
$$

Since $x, y$ were taken arbitrarily, then

$$
\omega_{d}(f, I \cap E) \leq \frac{\varepsilon}{2}+\omega_{d}\left(f_{n}, I \cap E\right)<\varepsilon+\omega_{d}\left(f_{n}, I \cap E\right) .
$$

For the same reason, one can infer that

$$
\omega_{d}\left(f_{n}, I \cap E\right) \leq \frac{\varepsilon}{2}+\omega_{d}(f, I \cap E)<\varepsilon+\omega_{d}(f, I \cap E) .
$$

We have proved that the inequality

$$
\left|\omega_{d}(f, I \cap E)-\omega_{d}\left(f_{n}, I \cap E\right)\right|<\varepsilon
$$

holds for each $n$ not less than $n_{0}$, which implies (10).

Applying Lemma 3.1 in the classical way, one can prove the following 


\section{ROBERT DROZDOWSKI}

TheOREM 3.2. If $(X, d)$ is a metric space with translation invariant metric and $\left(f_{n}\right)_{n=1}^{\infty}$ converges uniformly to $f$ with respect to metric $d$, where $f_{n}, f: E \rightarrow X$ for all $n$ from $\mathbb{N}$, then

$$
V_{d}(f, E) \leq \liminf _{n \rightarrow \infty} V_{d}\left(f_{n}, E\right) .
$$

Theorem 3.3. If $(X, d)$ is a complete metric space with translation invariant metric, then $d \widetilde{V} B G$ is complete metric space.

P r o of. Let $\left(f_{n}\right)_{n=1}^{\infty}$ be a Cauchy sequence in $d \widetilde{V} B G$ and $\varepsilon$ be a positive real number. There exists $n_{0}$ such that $V_{d}\left(f_{n}-f_{m}, E\right)<\varepsilon$ for $m, n$ not less than $n_{0}$. For each partition $\left\{I_{1}, \ldots, I_{k}\right\}$ from $\mathcal{R}(E)$, the inequality

$$
\sum_{i=1}^{k} \omega_{d}\left(f_{n}-f_{m}, I_{i} \cap E\right)<\frac{\varepsilon}{2}
$$

holds. Let us put

$$
F_{n}(x, y)=f_{n}(x)-f_{n}(y) \quad x, y \in E .
$$

By (2), the sequence $\left(F_{n}(x, y)\right)_{n=1}^{\infty}$ satisfies Cauchy's condition in a complete space $X$, so it converges to an element $a(x, y) \in X$. Let us denote by $F$ a function from $E \times E$ to $X$ such that $F(x, y)=a(x, y)$ for $x, y \in E$. We can define a function $f$ from $E$ to $X$ such that

$$
f(x)-f(y)=F(x, y) \quad \text { for each } x, y \text { from } E .
$$

It is sufficient to prove that $f$ belongs to $d \widetilde{V} B G$. According to (2), the sequence $\left(F_{n}\right)_{n=1}^{\infty}$ converges uniformly to a function $F$ with respect to metric $d$ on $E$. Using Lemma 3.1 and the inequality (2), one can get

$$
\sum_{i=1}^{k} \omega_{d}\left(f_{n}-f, I_{i} \cap E\right)<\varepsilon
$$

for any partition $\left\{I_{1}, \ldots, I_{k}\right\}$ from $\mathcal{R}(E)$. Hence, the sequence $\left(V_{d}\left(f_{n}-f, E\right)\right)_{n=1}^{\infty}$ converges to zero and, consequently, the sequence $\left(V_{d}\left(f_{n}, E\right)\right)_{n=1}^{\infty}$ satisfies Cauchy's condition in a complete space $\mathbb{R}$. Thus $\left(V_{d}\left(f_{n}, E\right)\right)_{n=1}^{\infty}$ is bounded, and by Theorem 3.2, $V_{d}(f, E)<\infty$. Finally, $f$ belongs to $d \widetilde{V} B G$.

Definition $3.1([7)$. It is said that a linear topological space $X$ is an $F$-space, if it is metrizable by a complete and translation invariant metric. Additionally, if $X$ is a locally convex $F$-space, then it is called a Fréchet space.

TheOREM 3.4. If $X$ is a linear space with translation invariant and complete metric $d$, which satisfies the condition of Theorem 3.1, then $d \widetilde{V} B G$ is an F-space. 


\section{ON THE GENERALIZED VARIATION}

P r o of. By Theorem 3.2, $d \widetilde{V} B G$ is complete and, from Theorem 3.1, it is a linear topological space. According to Definition 3.1, it is an $F$-space.

Theorem 3.5. Let $(X, d)$ be a metric space with translation invariant and complete metric d, where d satisfies the additional condition

- for each $\alpha$ from $[0,1]$ and $x \in X$, the inequality $d(\alpha x, 0) \leq \alpha d(x, 0)$ holds.

Then $d \widetilde{V} B G$ is a Fréchet space.

Proof. It is sufficient to show that $d \widetilde{V} B G$ is locally convex, since it is an $F$-space by Theorem 3.4, Denote by $\mathcal{B}$ the family

$$
\mathcal{B}=\left\{B^{\circ}(0, \varepsilon) \subset d \widetilde{V} B G: \varepsilon>0\right\} .
$$

We will prove that each of open balls from $\mathcal{B}$ is convex. Let us fix a positive real number $\varepsilon$, and take $\widetilde{f}, \widetilde{g}$ from $B^{\circ}(0, \varepsilon)$ and $\alpha, \beta$ from $[0,1]$ such that $\alpha+\beta=1$. Let $\left\{I_{1}, \ldots, I_{n}\right\}$ be an arbitrary partition of $E$ from $\mathcal{R}(E)$. If $x, y$ belong to $I_{i} \cap E$, then

$$
\begin{aligned}
d(\alpha f(x)+\beta g(x), \alpha f(y)+\beta g(y)) & =d(\alpha f(x)-\alpha f(y), \beta g(y)-\beta g(x)) \\
& \leq d(\alpha f(x)-\alpha f(y), 0)+d(0, \beta g(y)-\beta g(x)) \\
& \leq \alpha d(f(x), f(y))+\beta d(g(x), g(y)) \\
& \leq \alpha \omega_{d}\left(f, I_{i} \cap E\right)+\beta \omega_{d}\left(g, I_{i} \cap E\right),
\end{aligned}
$$

SO

$$
\omega_{d}\left(\alpha f+\beta g, I_{i} \cap E\right) \leq \alpha \omega_{d}\left(f, I_{i} \cap E\right)+\beta \omega_{d}\left(g, I_{i} \cap E\right)
$$

and, consequently,

$$
\begin{aligned}
\sum_{i=1}^{n} \omega_{d}\left(\alpha f+\beta g, I_{i} \cap E\right) & \leq \sum_{i=1}^{n} \alpha \omega_{d}\left(f, I_{i} \cap E\right)+\sum_{i=1}^{n} \beta \omega_{d}\left(g, I_{i} \cap E\right) \\
& \leq \alpha V_{d}(f, E)+\beta V_{d}(g, E)
\end{aligned}
$$

Thus

$$
V_{d}(\alpha f+\beta g, E) \leq \alpha V_{d}(f, E)+\beta V_{d}(g, E)<\alpha \varepsilon+\beta \varepsilon=\varepsilon .
$$

and finally, $\alpha \widetilde{f}+\beta \widetilde{g}$ belongs to $B^{\circ}(0, \varepsilon)$.

At the end of this section, we will define a new space, which will be denoted by the symbol $\Gamma \widetilde{W} B G(X)$. Recall that if $X$ is a linear topological space and $\Gamma \subset X^{\star}$, then $\Gamma \widetilde{W} B G(X)$ denotes the class of all maps from $E$ to $X$, which has a bounded weak variation in the restricted sense on $E$ with respect to subfamily $\Gamma$.

We start with the following 
TheOREm 3.6. If $X$ is a linear topological space, $\Gamma$ is a nonempty subset of $X^{\star}$, then the formula

$$
\sigma_{\Gamma}(f, g)=\min \{\sup \{V(\Lambda \circ(f-g), E): \Lambda \in \Gamma\}, 1\}
$$

defines a pseudometric in $\Gamma W B G(X)$.

Proof. It is clear that $\sigma_{\Gamma}(f, f)=0$. For $f, g, h$ from $\Gamma W B G(X)$ and any continuous linear functional $\Lambda$ over $X$ from $\Gamma$

$$
V(\Lambda \circ(f-g), E)=V(\Lambda \circ(g-f), E)
$$

and

$$
V(\Lambda \circ(f-g), E) \leq V(\Lambda \circ(f-h), E)+V(\Lambda \circ(h-g), E) .
$$

Due to the above remarks and the definition of $\sigma_{\Gamma}$ we have

$$
\sigma_{\Gamma}(f, g)=\sigma_{\Gamma}(g, f) \quad \text { and } \quad \sigma_{\Gamma}(f, g) \leq \sigma_{\Gamma}(f, h)+\sigma_{\Gamma}(h, g) .
$$

Now, following the above theorem, the relation " $\sim{ }_{\Gamma}$ " defined as

$$
f \sim_{\Gamma} g \Longleftrightarrow \sigma_{\Gamma}(f, g)=0
$$

is an equivalence relation in $\Gamma W B G(X)$. Then, the symbol $\Gamma \widetilde{W} B G(X)$ will denote the set of all equivalence classes determined by " $\sim{ }_{\Gamma}$ ". With respect to those remarks, if $X$ is a linear topological space, then the formula

$$
\widetilde{\sigma}(\widetilde{f}, \widetilde{g})=\sigma_{\Gamma}(f, g) \quad(\widetilde{f}, \widetilde{g} \in \Gamma \widetilde{W} B G(X))
$$

defines a translation invariant metric in $\Gamma \widetilde{W} B G(X)$.

Theorem 3.7. Let $X$ be a linear topological space and $\Lambda \in X^{\star}$. If $f_{n}, f: E \rightarrow X$ for each $n \in \mathbb{N}$ and $\left(f_{n}\right)_{n=1}^{\infty}$ converges uniformly to $f$ on $E$, then

$$
V(\Lambda \circ f, E) \leq \liminf _{n \rightarrow \infty} V\left(\Lambda \circ f_{n}, E\right) .
$$

P r o of. In the first step, we prove that

$$
\omega(\Lambda \circ f, I \cap E)=\lim _{n \rightarrow \infty} \omega\left(\Lambda \circ f_{n}, I \cap E\right)
$$

for each interval $I$ such that $I \cap E \neq \emptyset$. If $\varepsilon$ is a positive real number, then $\Lambda^{-1}((-\varepsilon, \varepsilon))$ is a neighborhood of 0 . By assumptions, there exists $n_{0} \in \mathbb{N}$ such that

$$
f_{n}(x)-f(x) \in \Lambda^{-1}\left(\left(-\frac{\varepsilon}{2}, \frac{\varepsilon}{2}\right)\right)
$$


for $x$ from $I \cap E$ and $n \geq n_{0}$. Owing to this,

$$
\begin{aligned}
|| \Lambda f_{n}(x)-\Lambda f_{n}(y)|-| \Lambda f(x)-\Lambda f(y)|| & \leq\left|\Lambda\left(f_{n}(x)-f(x)\right)+\Lambda\left(f(x)-f_{n}(y)\right)\right| \\
& \leq\left|\Lambda f_{n}(x)-\Lambda f(x)\right|+\left|\Lambda f_{n}(x)-\Lambda f(x)\right| \\
& <\varepsilon
\end{aligned}
$$

for arbitrary $x, y$ from $I \cap E$ and $n$ not less than $n_{0}$. Thus

$$
\left|\omega\left(\Lambda \circ f_{n}, I \cap E\right)-\omega(\Lambda \circ f, I \cap E)\right|<\varepsilon
$$

for $n \geq n_{0}$ and, consequently, (5) is true.

If $L=\liminf _{n \rightarrow \infty} V\left(\Lambda \circ f_{n}, E\right)$ and $L=\infty$ then (4) is satisfied. Now, let us assume that $L<\infty$ and choose an arbitrary partition $\left\{I_{1}, \ldots, I_{m}\right\}$ from $\mathcal{R}(E)$ and a positive real number $\eta$. There exists a subsequence $\left(f_{n_{k}}\right)_{k=1}^{\infty}$ such that

$$
V\left(\Lambda \circ f_{n_{k}}, E\right)<L+\eta \quad \text { for } \quad k \in \mathbb{N} \text {. }
$$

Then,

$$
\sum_{i=1}^{m} \omega\left(\Lambda \circ f_{n_{k}}, I_{i} \cap E\right)<L+\eta,
$$

and according to the previous part of the proof

$$
\sum_{i=1}^{m} \omega\left(\Lambda \circ f, I_{i} \cap E\right) \leq L+\eta .
$$

Since $\left\{I_{1}, \ldots, I_{m}\right\}$ and $\eta$ has chosen arbitrarily then

$$
V(\Lambda \circ f, E) \leq \liminf _{n \rightarrow \infty} V\left(\Lambda \circ f_{n}, E\right) .
$$

TheOREm 3.8. If $X$ is a linear topological space, $\Gamma$ is a nonempty subset of $X^{\star}$, $f_{n}, f: E \rightarrow X$ for $n \in \mathbb{N}$ and $\left(f_{n}\right)_{n=1}^{\infty}$ converges uniformly to $f$ on $E$, then

$$
\widetilde{\sigma}(\widetilde{f}, \widetilde{0}) \leq \liminf _{n \rightarrow \infty} \widetilde{\sigma}\left(\widetilde{f_{n}}, \widetilde{0}\right)
$$

Pr o of. By the last theorem, the inequality

$$
V(\Lambda \circ f, E) \leq \liminf _{n \rightarrow \infty} V\left(\Lambda \circ f_{n}, E\right)
$$

holds for each $\Lambda$ from $\Gamma$. Thus

$$
V(\Lambda \circ f, E) \leq \liminf _{n \rightarrow \infty} \sup \left\{V\left(\Lambda \circ f_{n}, E\right): \Lambda \in \Gamma\right\},
$$

so

$$
\sup \{V(\Lambda \circ f, E): \Lambda \in \Gamma\} \leq \liminf _{n \rightarrow \infty} \sup \left\{V\left(\Lambda \circ f_{n}, E\right): \Lambda \in \Gamma\right\}
$$


for each $\Lambda$ from $\Gamma$. Since

$$
\begin{aligned}
\min \{\sup \{V(\Lambda \circ f, E): \Lambda \in \Gamma\}, 1\} & \leq \\
& \min \left\{\liminf _{n \rightarrow \infty} \sup \left\{V\left(\Lambda \circ f_{n}, E\right): \Lambda \in \Gamma\right\}, 1\right\}
\end{aligned}
$$

and

$$
\begin{aligned}
& \min \left\{\liminf _{n \rightarrow \infty} \sup \left\{V\left(\Lambda \circ f_{n}, E\right): \Lambda \in \Gamma\right\}, 1\right\}= \\
& \liminf _{n \rightarrow \infty}\left(\min \left\{\sup \left\{V\left(\Lambda \circ f_{n}, E\right): \Lambda \in \Gamma\right\}, 1\right\}\right)
\end{aligned}
$$

then (6) and (7) give the required inequality.

From now on, for a family $\Gamma=\{\Lambda\}$, where $\Lambda$ is a functional from $X^{\star}$, we will write $\Lambda \widetilde{W} B G$ instead of $\Gamma \widetilde{W} B G$.

TheOREM 3.9. If $(X, d)$ is a metric space with translation invariant metric $d$ and $\Lambda$ is a linear functional over $X$ such that there exists a positive real number $M>0$ that is $M d(x, 0) \leq|\Lambda x|$ for each $x$ from $X$, then $\Lambda \widetilde{W} B G(X)$ is a complete space.

Pr o of. Let $\left(\widetilde{f_{n}}\right)_{n=1}^{\infty}$ be a Cauchy sequence in $\Lambda \widetilde{W} B G(X)$.

Then, for a positive real number $\varepsilon$ there exists a positive integer $n_{0}$ such that

$$
V\left(\Lambda \circ\left(f_{n}-f_{m}\right), E\right)<\varepsilon
$$

i.e.,

$$
\sum_{i=1}^{k} \omega\left(\Lambda \circ\left(f_{n}-f_{m}\right), I_{i} \cap E\right)<\varepsilon
$$

for $m, n$ not less than $n_{0}$ and for each sequence $\left\{I_{1}, \ldots, I_{k}\right\}$ from $\mathcal{R}(E)$.

Let us denote

$$
F_{n}(x, y)=f_{n}(x)-f_{n}(y) \quad x, y \in E .
$$

If $\left\{I_{1}, \ldots, I_{k}\right\}$, where $k \in \mathbb{N}$, is a partition of $E$ from $\mathcal{R}(E)$, then

$$
\sum_{i=1}^{k} \omega\left(\Lambda \circ\left(f_{n}-f_{m}\right), I_{i} \cap E\right)<\varepsilon,
$$

which implies that

$$
\left|\Lambda\left(F_{n}(x, y)\right)-\Lambda\left(F_{m}(x, y)\right)\right|<\varepsilon
$$

where $n, m \geq n_{0}$. Whence $\left(\Lambda\left(F_{n}(x, y)\right)\right)_{n=1}^{\infty}$ is a Cauchy sequence in a complete space $\Phi$ for each $x, y$ from $E$. Let us denote by $f$ a function from $E$ to $X$ such that

$$
\Lambda(f(x)-f(y)):=\lim _{n \rightarrow \infty} \Lambda\left(F_{n}(x, y)\right)
$$




\section{ON THE GENERALIZED VARIATION}

One can observe that if $x, y \in E$, then the inequality

$$
\left|\Lambda\left(F_{n}(x, y)\right)-\Lambda\left(F_{m}(x, y)\right)\right| \leq \varepsilon
$$

holds, and from properties of $\Lambda$

$$
M d\left(F_{n}(x, y)-(f(x)-f(y)), 0\right) \leq\left|\Lambda\left(F_{n}(x, y)\right)-\Lambda(f(x)-f(y))\right| \leq \varepsilon
$$

for each $x, y$ from $E$ and $n$ not less than $n_{0}$. It implies that $\left(F_{n}\right)_{n=1}^{\infty}$ converges uniformly to the map $F(x, y)=f(x)-f(y)$. By Theorem 3.6.

$$
V(\Lambda \circ f, E) \leq \liminf _{n \rightarrow \infty} V\left(\Lambda \circ f_{n}, E\right),
$$

whence $V(\Lambda \circ f, E)$ is finite. As a result we get that $f$ belongs to $\Lambda \widetilde{W} B G(X)$. In addition,

$$
V\left(\Lambda \circ\left(f_{n}-f\right), E\right) \leq \varepsilon
$$

so

$$
\lim _{n \rightarrow \infty} \tilde{\sigma}\left(\widetilde{f_{n}}, \widetilde{f}\right)=0
$$

and the proof is complete.

Theorem 3.10. $\widetilde{\Gamma} B G(X)$ is a locally convex space for each subfamily $\Gamma$ of $X^{\star}$.

P r o of. By simple calculus one can show that $B^{\circ}\left(0, \frac{1}{n}\right)$ are convex for each $n$ from $\mathbb{N}$, so the proof is omitted.

From Theorem 3.9 and Theorem 3.10 it immediately follows

Theorem 3.11. $\Lambda \widetilde{W} B G(X)$ is a Fréchet space for each space $X$ and functional $\Lambda$ from $X^{\star}$, which satisfies the conditions of Theorem 3.9 .

\section{Comparison of spaces: $d V B G, d V B G_{\star}, \Gamma W B G(X)$, $\Gamma W B G_{\star}(X)$}

In this section, we will try to establish the connections between $d V B G$ and $\Gamma W B G(X)$, where $(X, d)$ is a linear and metric space with translation invariant metric $d$ and $\Gamma$ is a subset of $X^{\star}$. Immediately from definitions of $d V B G, d V B G_{\star}$, it follows that $d V B G \subset d V B G_{\star}$. If $X$ is a linear topological space then it is clear that $\Gamma W B G(X) \subset \Gamma W B G_{\star}(X)$ for each subfamily $\Gamma$ of $X^{\star}$.

From now on, we will consider only such metric spaces, for which every bounded subset in the usual sense in $X$ is bounded in the sense of linear topological spaces. 
TheOREM 4.1. Let $(X, d)$ be a metric linear space with a translation invariant metric, $\Gamma$ be a nonempty subfamily of $X^{\star}$. If for each bounded subset $U$ of $X$ the set $U_{d}$ of the form

$$
U_{d}=\left\{\frac{x}{d(x, 0)}: x \in U \backslash\{0\}\right\}
$$

is bounded, then $d V B G \subset \Gamma W B G(X)$ and $d V B G_{\star} \subset \Gamma W B G_{\star}(X)$.

P r o of. We only prove the first inclusion, since the second inclusion is a simple consequence of the first one.

Let us choose $f$ from $d V B G, \Lambda$ from $\Gamma$ and a partition $\left\{I_{1}, \ldots, I_{n}\right\}$, where $n \in \mathbb{N}$, from $\mathcal{R}(E)$. Since $\Lambda$ is a continuous and linear functional in metric linear space $X$, so it is bounded, that means $\Lambda(W)$ is bounded in $\Phi$ for each bounded subset $W$ of $X$. Since $f$ belongs to $d V B G$, then, by Theorem 2.2, $f(E)$ is bounded in $X$ in the usual sense. By assumption of $X, f(E)$ is bounded in $X$ and hence $f(E)-f(E)$ is a bounded subset of $X$, too. Then

$$
A=\left\{\frac{f(x)-f(y)}{d(f(x), f(y))}: f(x) \neq f(y) \wedge x, y \in E\right\}
$$

is bounded in $X$, so there exists a positive real number $M$ such that

$$
|\Lambda f(x)-\Lambda f(y)| \leq M d(f(x), f(y))
$$

for all vectors from $A$. If $x, y$ belong to $I_{i} \cap E$, then

$$
|\Lambda f(x)-\Lambda f(y)|=|\Lambda(f(x)-f(y))| \leq M d(f(x), f(y)) \leq M \omega_{d}\left(f, I_{i} \cap E\right) .
$$

Thus

$$
\omega\left(\Lambda \circ f, I_{i} \cap E\right) \leq M \omega_{d}\left(f, I_{i} \cap E\right)
$$

and

$$
\sum_{i=1}^{n} \omega\left(\Lambda \circ f, I_{i} \cap E\right) \leq M \cdot \sum_{i=1}^{n} \omega_{d}\left(f, I_{i} \cap E\right) \leq M \cdot V_{d}(f, E) .
$$

Since $\left\{I_{1}, \ldots, I_{n}\right\}$ and $\Lambda$ have been chosen in an arbitrary way, so

$$
V(\Lambda \circ f, E) \leq M \cdot V_{d}(f, E)
$$

and, consequently, $f \in \Gamma W B G_{\star}(X)$.

TheOREM 4.2. If $\left(X,\|\cdot\|_{X}\right)$ is a normed space, $\Gamma$ is a nonempty subfamily of $X^{\star}$, then

$$
d V B G \subset \Gamma W B G(X) \text { and } d V B G_{\star} \subset \Gamma W B G_{\star}(X),
$$

where $d(x, y)=\|x-y\|_{X}$ for each $x, y$ from $X$.

Let us see that the inclusions in Theorem 4.1 might be proper. 


\section{ON THE GENERALIZED VARIATION}

EXAMPLE 4.1. Let us consider the space $L^{p}([0,1])$ of all measurable functions $f$ from $[0,1]$ to $\mathbb{C}$ with respect to Lebesgue measure $\lambda$ and such that $\int_{0}^{1}|f|^{p} \mathrm{~d} \lambda<\infty$, where $p \in(0,1)$. Then the function $d$ defined in the following way

$$
d(f, g)=\int_{0}^{1}|f(x)-g(x)|^{p} \mathrm{~d} \lambda, \quad f, g \in L^{p}([0,1])
$$

is a metric in $L^{p}([0,1])$ (for details, see [7]).

For a real number $\eta$, let $g_{\eta}$ denote the functions from $[0,1]$ into $[0,1]$, where

$$
g_{\eta}(x)=\eta x \quad \text { and } \quad x \in[0,1] .
$$

If $f: \mathbb{R} \rightarrow L^{p}([0,1])$, where $f(\eta)=g_{\eta}$, then $\omega_{d}(f,[n, n+1]) \geq 1$ for all $n$ from $\mathbb{N}$. Hence $V(f, \mathbb{R})=\infty$, that means $f$ does not belong to $d V B G_{\star}$. One can show that $W B G_{\star}\left(L^{p}([0,1])\right)$ consists of all mappings from $[0,1]$ to $X$.

Definition $4.1\left([4)\right.$. It is said that the metrics $d_{1}$ and $d_{2}$ are uniformly equivalent in $X$ if there exist positive real numbers $\alpha$ and $\beta$ such that

$$
\alpha d_{1}(x, y) \leq d_{2}(x, y) \leq \beta d_{1}(x, y)
$$

for each $x, y$ from $X$.

TheOREM 4.3. If $X$ is a linear space and $d, d^{\prime}$ are uniformly equivalent and translation invariant metrics in $X$, then

$$
\begin{aligned}
d V B G & =d^{\prime} V B G, \quad W B G((X, d))=W B G\left(\left(X, d^{\prime}\right)\right), \\
d V B G_{\star} & =d^{\prime} V B G_{\star}, \quad W B G_{\star}((X, d))=W B G_{\star}\left(\left(X, d^{\prime}\right)\right) .
\end{aligned}
$$

Proof. By assumptions, there exist some positive real numbers $M_{1}$ and $M_{2}$ such that

$$
M_{1} d(x, y) \leq d^{\prime}(x, y) \leq M_{2} d(x, y)
$$

for all $x, y$ from $X$. If $I$ is a closed interval such that $E \cap I \neq \emptyset$, then

$$
M_{1} \omega_{d}(f, I \cap E) \leq \omega_{d^{\prime}}(f, I \cap E) \leq M_{2} \omega_{d}(f, I \cap E) .
$$

By the above observation, we can obtain

$$
M_{1} V_{d}(f, E) \leq V_{d^{\prime}}(f, E) \leq M_{2} V_{d}(f, E),
$$

that means $f \in d V B G$ if $f \in d^{\prime} V B G$.

Let us assume that $f$ belongs to $W B G((X, d))$. If $\Lambda$ is a continuous functional over $X$ with respect to metric $d^{\prime}$ then, by assumptions, it is also continuous over $X$ with respect to metric $d$, so $V(\Lambda \circ f, E)<\infty$ for all linear functionals $\Lambda$, which are continuous with respect to metric $d^{\prime}$. Hence,

$$
W B G((X, d)) \subset W B G\left(\left(X, d^{\prime}\right)\right) .
$$

The inverse inclusion can be proved in the same way. 


\section{ROBERT DROZDOWSKI}

\section{REFERENCES}

[1] BRUCKNER, A. M.: Differentation of Real Functions. Lecture Notes in Math., Vol. 659, Springer-Verlag, Berlin, 1978.

[2] CIEMNOCZOŁOWSKI, J.-ORLICZ, W.: Inclusions theorem for classes of functions of generalized bounded variation, Ann. Soc. Math. Pol., Ser. I., Commentat. Math. 24 (1984), 181-194.

[3] DROZDOWSKI, R.: On the structure of some subsets of interval function of bounded $S C(\Lambda)$-variation, Tatra Mt. Math. Publ. 37 (2007), 1-9.

[4] ENGELKING, R.: General Topology. PWN, Warszawa, 1977.

[5] PERLMAN, S.-WATERMAN, D.: Some remarks on functions of $\Lambda$-bounded-variation, Proc. Amer. Math. Soc. 74 (1979), 113-118.

[6] ROLEWICZ, S.: Metric Linear Spaces. PWN, Warszawa, 1984.

[7] RUDIN, W.: Functional Analysis (2nd ed.), McGraw Hill Book Company, New York, 1991.

[8] SAKS, S.: Theory of the Integral. G. E. Stechert \& Co., New York, 1937.

[9] THOMSON, B.: Real Functions. Lecture Notes in Math., Vol. 1170, Springer-Verlag, Berlin, 1985.

Received November 17, 2007

Institute of Mathematics

Academia Pomeraniensis

Arciszewskiego 22b

PL-76-200 Stupsk

POLAND

E-mail: r.drozdowski@wp.pl 